\title{
A Three-objective User Equilibrium Model: Time Surplus Maximisation under Uncertainty
}

\author{
Judith Y.T. Wang \\ School of Civil Engineering \& Institute for Transport Studies \\ University of Leeds, Woodhouse Lane, Leeds LS2 9JT, UK \\ email: j.y.t.wang@leeds.ac.uk \\ Matthias Ehrgott* \\ Department of Management Science, Lancaster University Management School \\ Bailrigg, Lancaster LA1 4YX, UK \\ email: m.ehrgott@lancaster.ac.uk
}

\begin{abstract}
In this paper, we propose a user equilibrium model considering the three most important factors influencing route choice behaviour in a road network, namely, travel time, travel time reliability and monetary cost. We further develop the time surplus maximisation bi-objective user equilibrium (TSmaxBUE) model and incorporate the concept of travel time budget to model how users might react to uncertainty induced by day-to-day variability in travel time caused by traffic incidents. This results in a three-objective user equilibrium model, which has a possibly infinite set of equilibrium flows. To compute equilibrium flows, we introduce time budget surplus (TBS) defined as the maximum travel time a user is willing to spend minus the actual time budget required for a desired level of travel time reliability. At equilibrium, for each origin-destination (O-D) pair, all individuals are travelling on the path with the highest TBS value among all the efficient paths between this O-D pair. This becomes a time budget surplus maximisation three-objective user equilibrium model (TBSmaxTUE). We show that the TBSmaxTUE model is a special case of three-objective user equilibrium considering minimisation of expected travel time, travel time variance and toll (monetary cost) as objectives. We illustrate the model and our results on a small network.
\end{abstract}

Keywords: Traffic assignment; route choice; equilibrium problem; multi-objective optimisation; travel time reliability.

\section{Introduction}

It is well known that the three most important criteria affecting route choice behaviour are travel time, travel time reliability and monetary cost. This observation is drawn from numerous studies in the

\footnotetext{
${ }^{*}$ Corresponding author. Tel.: +44113343 3259 ; Fax: +44 1133433265.
} 
literature. From a route choice survey, Abdel-Aty et al. (1995) identified the three most important factors to be: (1) shorter travel time (ranked as the first reason by $40 \%$ of respondents); (2) travel time reliability (32\%); and (3) shorter distance (31\%). Note that although the effect of monetary cost was not considered explicitly in this study, the third most important factor, i.e. distance, is directly related to vehicle operating cost for the trip. From numerous road pricing studies in the US (e.g. Lam \& Small 2001, Liu et al. 2004, Brownstone \& Small 2005) and in Australia (Li et al. 2010), it is also clear that the value of reliability (VOR) estimates, when travel time is represented by its median and unreliability of travel time as the difference between the 90th percentile and the median of travel time, can be as high as the value of time (VOT). For some user groups, VOR was found to be even higher than VOT, e.g. the VOR for women is $39.5 \%$ higher than the VOT in the best fitted model in Lam \& Small (2001). In order to model route choice behaviour realistically, there is no doubt we should consider the influence of all these three factors.

Network equilibrium models have been studied for decades since the discovery of Wardrop's principles. Combinations of some of the three most important route choice factors mentioned above have also been considered in many studies (e.g. Florian 2006, Lo et al. 2006, Watling 2006). Florian (2006) summarised the two approaches employed in practice, when toll and travel time are considered: (1) models based on generalised cost path choice; and (2) models based on explicit choice of tolled facilities. These two approaches follow the principles of the two classical traffic assignment models in the literature, namely, the user equilibrium (UE) model and the stochastic user equilibrium (SUE) model.

Lo et al. (2006) formulated a multi-class equilibrium model by considering travel time and travel time reliability, combined in a single objective as minimising travel time budget, which is defined as the expected travel time plus a travel time margin (or buffer time), with the travel time margin being dependent on the level of risk aversion of each user class. Watling (2006) proposed a late arrival penalised UE (LAPUE) which assumes users minimise a composite path disutility, incorporating the generalised cost plus a late arrival penalty. Watling (2006) assumes that travellers make their route choice decision with a longest possible travel time in mind for their journey. If this is exceeded, the inconvenience incurred will be modelled by the penalty component of the disutility function. In principle, Watling's and Lo et al.'s models can be applied in a manner to consider all three factors, with toll/monetary cost and travel time considered as generalised travel time, and travel time reliability in the penalty component or travel time and travel time reliability in the time budget plus the time equivalent of monetary cost, respectively.

All existing network equilibrium models share one common feature, i.e. all the factors considered are combined into a single objective. As discussed in Wang \& Ehrgott (2013), the linear combination of time and cost, for instance, implies that a certain class of efficient paths will have zero flow at the equilibrium solution, and hence might not be able to model route choice behaviour realistically. Similarly, Wang et al. (2014) show that Lo et al.'s and Watling's models might miss out some rational route choices in a similar manner.

To address this issue, Wang \& Ehrgott (2013) propose a bi-objective approach to overcome the 
problem, without assuming the two objectives of travel time and travel time reliability being additive as in Dial (1996). Wang \& Ehrgott introduce the concept of time surplus maximisation to model route choice behaviour in a tolled road network considering two objectives: (1) minimise travel time; and (2) minimise toll cost. Time surplus is defined as the maximum time users are willing to spend minus the actual travel time. All users would want to maximise time surplus based on the two objectives. This becomes the time surplus maximisation (bi-objective) equilibrium model (TSmaxBUE). At equilibrium, only the paths with the maximal time surplus will have positive flow. Differences in preferences among travellers between each origin-destination (O-D) pair are modelled by indifference curves, which represent the maximum time a user is willing to spend for a given toll. The TSmaxBUE model also satisfies the condition for bi-objective user equilibrium as defined in Wang et al. (2010), whereby no user can decrease travel time, or toll, or both without worsening the other and vice versa. As demonstrated in Wang \& Ehrgott (2013), the TSmaxBUE model is a proper generalisation of the UE model with additive generalised cost functions.

On the other hand, Wang et al. (2014) consider travel time and travel time reliability with a biobjective approach. Wang et al. propose a general network equilibrium model, travel time reliability bi-objective user equilibrium (TTR-BUE) model, considering two objectives: (1) minimise travel time; and (2) minimise travel time variability. Wang et al. prove that existing travel time reliability models, including Lo et al.'s and Watling's models, as well as their bi-objective counterparts are special cases of TTR-BUE. For example, the bi-objective version of Lo et al.'s model has the two objectives (1) minimise expected travel time and (2) minimise travel time budget. This is a special case of TTR-BUE.

In this paper, we propose the first multi-objective network equilibrium model considering all the three most important objectives affecting route choice. We further develop the TSmaxBUE model from Wang \& Ehrgott (2013), incorporating findings from Wang et al. (2014), to include the consideration of travel time reliability. We note that multi-objective user equilibrium models in general admit an infinite number of equilibrium flows. Despite the drawback of losing uniqueness of equilibrium solutions, this allows the verification that observed flows are consistent with assumptions on the user objectives, independent of any parameters used to convert the multiple objectives into a single cost function. In order to be able to compute the set of equilibrium flows, we must, however, first address the question of computing single equilibrium flows. In this paper we propose a model that makes this possible.

The rest of the paper is organised as follows. Section 2 introduces notation and presents the notion of user equilibrium models of travel assignment. We review the concepts of generalised cost, travel time reliability and travel time budget user equilibrium. We also state a small three-link example that we use throughout the paper to illustrate results. In Section 3, we give a general definition of multi-objective user equilibrium and prove some general results. Section 4 is dedicated to a summary of the travel time surplus maximisation bi-objective user equilibrium model. In Section 5, the main results of the paper on a generalisation of the TSmaxBUE model and the incorporation of travel time reliability through the travel time budget equilibrium model are discussed and the relationships of this 
time budget surplus maximisation model to multi-objective user equilibrium models are investigated. We also present an NCP formulation of the time budget surplus maximisation model. Section 6 explores the new model on the three-link example in detail and Section 7 concludes the paper with some general comments and suggested avenues for future research.

\section{User Equilibrium}

In this section, we briefly review equilibrium models of traffic assignment and introduce notation, as well as an example, that are used throughout the paper.

\subsection{Traditional Traffic Assignment Models}

Let $G=(N, A)$ denote a network, where $N$ is a finite set of $|N|$ nodes and $A \subset N \times N$ is a set of $|A|$ arcs or links. Moreover, let $Z \subset N \times N$ be a set of origin-destination pairs (O-D pairs) and for all $p \in Z$, let $D_{p}$ denote the demand for travel between the origin and destination of O-D pair $p$. Equilibrium models attempt to determine the amount of traffic $f_{a}$ on all links $a \in A$ under some assumptions on the behaviour of road users. One of these assumptions is that road users choose a path $k^{*}$ between their origin and destination that minimises a non-negative cost function $C_{k}$ :

$$
k^{*} \in \operatorname{argmin}\left\{C_{k}: k \in K_{p}\right\}
$$

where $K_{p}$ is the set of all simple paths from the origin of O-D pair $p$ to its destination.

Let $\delta_{a}^{k}$ be an indicator with $\delta_{a}^{k}=1$ if and only if link $a$ is contained in path $k$ and 0 otherwise. Then $f_{a}=\sum_{p \in Z} \sum_{k \in K_{p}} \delta_{a}^{k} F_{k}$, where $F_{k}$ is the flow on path $k \in K_{p}$. The $\operatorname{cost} C_{k}(\mathbf{F})$ of path $k$ may depend on the entire vector $\mathbf{F}=\left(F_{1}, \ldots, F_{|K|}\right)$ of flows on all paths $k \in K:=\cup_{p \in Z} K_{p}$. The user equilibrium condition of Wardrop's first principle states that the costs of all used paths are equal and not greater than the cost which would be experienced by a single user on any unused route.

Let $U_{p}:=\min _{k \in K_{p}} C_{k}(\mathbf{F})$ denote the minimum cost of any path for O-D pair $p \in Z$. Then, following e.g. Florian \& Hearn (1995), the user equilibrium condition can be written mathematically as follows: Path flow vector $\mathbf{F}^{*}$ is an equilibrium flow if $\mathbf{F}^{*}$ satisfies conditions (1) - (5):

$$
\begin{aligned}
F_{k}^{*}\left(C_{k}\left(\mathbf{F}^{*}\right)-U_{p}\right) & =0 \quad \text { for all } k \in K_{p} \text { and all } p \in Z, \\
C_{k}\left(\mathbf{F}^{*}\right)-U_{p} & \geq 0 \quad \text { for all } k \in K_{p} \text { and all } p \in Z, \\
\sum_{k \in K_{p}} F_{k}^{*}-D_{p} & =0 \quad \text { for all } p \in Z, \\
F_{k}^{*} & \geq 0 \quad \text { for all } k \in K, \\
U_{p} & \geq 0 \quad \text { for all } p \in Z .
\end{aligned}
$$

Equation (1) states that if flow on path $k$ is positive then the cost $C_{k}\left(\mathbf{F}^{*}\right)$ has to be minimal, whereas if $C_{k}\left(\mathbf{F}^{*}\right)>U_{p}$ then the flow on path $k$ must be 0 . Equation (2) says that the costs of all paths in $K_{p}$ are greater than or equal to the minimum $U_{p}$. Equation (3) guarantees that demand is 
satisfied, whereas equations (4) and (5) postulate non-negativity of flow and cost. Note that For future use, let us introduce

$$
\Omega:=\{\mathbf{F}: \mathbf{F} \text { satisfies }(3)-(4)\}
$$

to denote the set of all feasible path flow vectors $\mathbf{F}$.

Existence of a solution of the network equilibrium model (1) - (5) is guaranteed if the path cost functions $C_{k}(\mathbf{F})$ are all positive and continuous. If, in addition, the vector of path cost functions $C: \mathbb{R}_{|K|} \rightarrow \mathbb{R}_{|K|}$ is strictly monotone, then equilibrium link flows are unique, see e.g. Smith (1979) for additive cost functions and Gabriel \& Bernstein (1997) for non-additive cost functions.

In this paper, we will consider equilibrium problems with multiple user classes. We use an index $m \in M$ to indicate user class. Equilibrium models with multiple user classes split the demand $D_{p}$ for each OD pair $p$ into separate demands $D_{p m}$ for each user class, hence $D_{p}=\sum_{m \in M} D_{p m}$. Consequently, flow vector $\mathbf{F}$ records flow $F_{k m}$ for each path $k$ and each user class $m, U_{p m}$ denotes the minimum cost of a path between OD pair $p$ and user class $m$ and path cost functions $C_{k m}$ will differ by user class. The equilibrium conditions (1) - (5) need to be satisfied for each user class.

\subsection{Travel Time, Generalised Cost and Generalised Time}

The most important cost function is travel time. In this paper, we use the common Bureau of Public Roads (1964) function to model the relation between travel time and traffic flow on any link $a \in A$, i.e.

$$
t_{a}\left(f_{a}\right)=t_{a}^{0}\left[1+\beta\left(\frac{f_{a}}{C_{a}}\right)^{n}\right]
$$

where $t_{a}^{0}$ is the free-flow travel time on link $a, C_{a}$ is the practical capacity of link $a$ in vehicles per time unit, and $\beta, n$ are function parameters. If the cost function $C_{k}$ considered in (1) - (5) is path travel time, then

$$
C_{k}(\mathbf{F})=T_{k}(\mathbf{F}):=\sum_{a \in k} t_{a}\left(f_{a}\right)
$$

for all $k \in K$.

The best known algorithm used to solve conventional traffic assignment is the Frank-Wolfe algorithm (Frank \& Wolfe 1956) but many others such as path equilibration (Dafermos \& Sparrow 1969), gradient projection (Jayakrishnan et al. 1994), projected gradient (Florian et al. 2009), and other methods (e.g. Bar-Gera (2010)) have been proposed, see e.g. Perederieieva et al. (2015).

Many researchers have suggested more general cost functions than travel time, see e.g. Chen et al. (2010), Larsson et al. (2002). Most often $C_{k}(\mathbf{F})$ takes the form of a generalised cost function that incorporates a linear combination of travel time and a monetary component (Dial 1996, Leurent 1993). An additive generalised cost function is of the form

$$
C_{k}(\mathbf{F})=M_{k}(\mathbf{F})+\alpha T_{k}(\mathbf{F})
$$

where $M_{k}(\mathbf{F})$ is the monetary cost associated with path $k$. This may be composed of different factors such as toll cost and vehicle operating costs. In addition, $\alpha$ is a value of time, i.e. it converts the 
travel time $T_{k}(\mathbf{F})$ into a monetary value. To solve traffic assignment problems with generalised cost function (9), one can apply the same algorithms as for conventional traffic assignment, depending on the properties of function $M_{k}(\mathbf{F})$. We note that some authors also consider (9) with a continuous distribution of $\alpha$, (Dial 1996, Leurent 1993).

Larsson et al. (2002) on the other hand considers a nonlinear "generalised time" function of the form

$$
C_{k}(\mathbf{F})=T_{k}(\mathbf{F})+G_{k}\left(M_{k}(\mathbf{F})\right),
$$

where $G_{k}: \mathbb{R} \rightarrow \mathbb{R}$ is a non-negative increasing function, the time-equivalent of money.

\subsection{Travel Time Reliability and Travel Time Budget Equilibrium}

To model the influence of travel time and travel time reliability on route choice behaviour, there are two main theoretical frameworks, as categorised in Li et al. (2010) and Carrion \& Levinson (2012), namely, the mean-variance model (Jackson \& Jucker 1982) and the scheduling model (Small 1982). Reliability based UE models were further developed based on these two concepts by Lo et al. (2006) and Watling (2006), respectively.

In this paper, we are interested in travel time variability due to variations in link capacity caused by day-to-day incidents. We assume that link capacity distributions are independent. To model the influence of such variability of travel time, Lo et al. (2006) formulated a multi-class equilibrium model considering the minimisation of travel time budget, defined as the expected travel time plus a travel time margin (or buffer time), with the travel time margin being dependent on the level of risk aversion of each user class, as shown in Equation (11),

$$
B_{k m}(\mathbf{F})=E\left(T_{k}(\mathbf{F})\right)+\lambda_{m} \sigma_{T_{k}(\mathbf{F})}
$$

for all $k \in K_{p}, m \in M$, and $p \in Z$, where $B_{k m}(\mathbf{F})$ is the travel time budget; $T_{k}(\mathbf{F})$ is the random variable of travel time on route $k$ between origin and destination of OD pair $p ; E\left(T_{k}(\mathbf{F})\right)$ and $\sigma_{T_{k}}(\mathbf{F})$, respectively, are the mean and standard deviation of $T_{k}(\mathbf{F}) . \lambda_{m}$ is a parameter associated with the level of risk aversion of user class $m$. We adopt the concept of travel time budget as a mechanism to deal with travel time variability in this paper, because we focus on modelling the travel behaviour of road users in response to the day-to-day variations in travel time induced by disruptions on a minor scale, caused by traffic incidents. We, therefore, adopt the results from Lo \& Tung (2003), summarised as follows for completeness.

Assuming that link capacity follows a uniform distribution, defined by an upper bound (the design capacity) and a lower bound (the worst-degraded capacity), which is a fraction, $\phi_{a}$, of the design capacity, $\bar{c}_{a}$, i.e.

$$
C_{a} \sim U\left(\phi_{a} \bar{c}_{a}, \bar{c}_{a}\right),
$$

Lo \& Tung (2003) have derived that, due to the central limit theorem for paths with many links, the 
path travel time is normally distributed with mean and standard deviation that can be written as

$$
\begin{aligned}
E\left(T_{k}\right) & =\sum_{a}\left[\delta_{a}^{k} \cdot E\left(t_{a}\right)\right] \\
\sigma_{T_{k}} & =\sqrt{\sum_{a}\left[\delta_{a}^{k} \cdot \operatorname{var}\left(t_{a}\right)\right]} .
\end{aligned}
$$

By applying the assumption of uniformly distributed arc capacity as expressed in Equation (12), the mean and standard deviation of the route travel time distribution are

$$
\begin{gathered}
E\left(T_{k}\right)=\sum_{a}\left\{\delta_{a}^{k} \cdot\left[t_{a}^{0}+\beta t_{a}^{0} f_{a}^{n} \frac{1-\phi_{a}^{1-n}}{\bar{c}_{a}^{n}\left(1-\phi_{a}\right)(1-n)}\right]\right\}, \\
\sigma_{T_{k}}=\sqrt{\sum_{a}\left[\delta_{a}^{k} \cdot \beta^{2}\left(t_{a}^{0}\right)^{2} f_{a}^{2 n}\left\{\frac{1-\phi_{a}^{1-2 n}}{\bar{c}_{a}^{2 n}\left(1-\phi_{a}\right)(1-2 n)}-\left[\frac{1-\phi_{a}^{1-n}}{\bar{c}_{a}^{n}\left(1-\phi_{a}\right)(1-n)}\right]^{2}\right\}\right]} .
\end{gathered}
$$

The travel time budget model of Lo et al. (2006) is a multi-user class equilibrium model which considers both the expected travel time $E\left(T_{k}\right)$ and the variability of travel time, as measured by $\sigma_{T_{k}}$ with users in class $m$ minimising their travel time budget $B_{k}=E\left(T_{k}\right)+\lambda_{m} \sigma_{T_{k}}$. Mathematically, $\lambda_{m}$ can be related to the probability $\rho_{m}$ that a trip arrives within the travel time budget,

$$
P\left\{T_{k} \leqslant B_{k}=E\left(T_{k}\right)+\lambda_{m} \sigma_{T_{k}}\right\}=\rho_{m} .
$$

After rearranging (17), we have

$$
P\left(S_{T_{k}}=\frac{T_{k}-E\left(T_{k}\right)}{\sigma_{T_{k}}} \leqslant \lambda_{m}\right)=\rho_{m} .
$$

Note that $S_{T_{k}}$ on the left hand side of Equation (18) is the standard normal variate of $T_{k}, S_{T_{k}} \sim$ $N(0,1)$.

To summarise, the path cost functions that we shall use in this paper are

$$
\begin{aligned}
T_{k}(\mathbf{F}):=\sum_{a \in k} t_{a}\left(f_{a}\right) ; \text { travel time on path } k ; \\
E\left(T_{k}(\mathbf{F})\right) \quad \text { defined by }(15) ; \text { expected path travel time on path } k ; \\
\sigma_{T_{k}(\mathbf{F})} \quad \text { defined by }(16) ; \text { standard deviation of path travel time on path } k ; \\
B_{k m}(\mathbf{F}):=E\left(T_{k}(\mathbf{F})\right)+\lambda_{m} \sigma_{T_{k}}(\mathbf{F}) ; \text { path travel time budget of user class } m \text { on path } k ; \\
\tau_{k}(\mathbf{F}):=\sum_{a \in k} \tau_{a} ; \text { toll on path } k ; \\
M_{k}(\mathbf{F}):=\sum_{a \in k} m_{a} ; \text { monetary cost on path } k .
\end{aligned}
$$

Notice that we assume that toll and monetary cost in general are independent of flows and that only the time budget $B_{k m}$ varies by user class through the risk aversion parameter $\lambda_{m}$. 


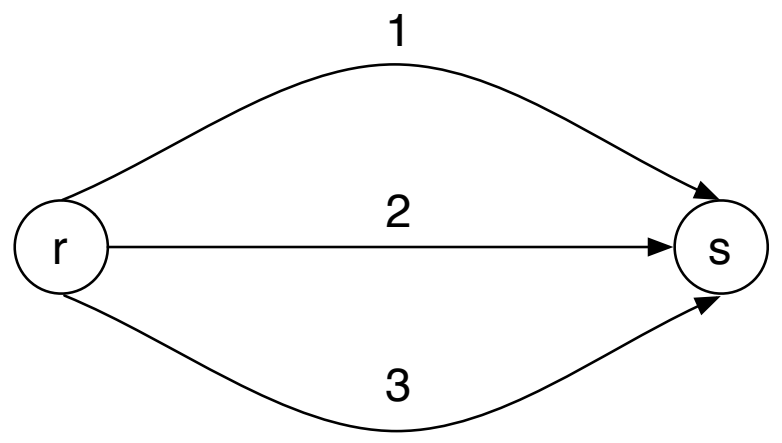

Figure 1: A three-link example network.

\subsection{A Three-link Example}

We will demonstrate the results of our research using a small three-link network, which is shown in Figure 1, where the link parameters are specified in Table 1. The parameters of the travel time function, Equation (7), are $\beta=0.15$ and $n=4$. The total demand is assumed to be fixed at 15,000 vehicles per hour. The free flow travel time $t_{a}^{0}$, capacity $C_{a}$, reliability parameter $\phi_{a}$ and two values of tolls $\tau_{a}^{1}$ and $\tau_{a}^{2}$ that we use later are summarised in Table 1 . We note that we shall use toll as the only monetary cost. Further parameters will be specified as necessary in the context of the examples illustrating specific results.

Table 1: Route characteristics of the three-link network.

\begin{tabular}{llrrrrrr}
\hline $\begin{array}{l}\text { Route } \\
a\end{array}$ & Type & $\begin{array}{r}\text { Distance } \\
(\mathrm{km})\end{array}$ & $\begin{array}{r}t_{a}^{0} \\
(\mathrm{mins})\end{array}$ & $\begin{array}{r}C_{a} \\
(\mathrm{veh} / \mathrm{hr})\end{array}$ & $\phi_{a}$ & $\tau_{a}^{1}$ & $\tau_{a}^{2}$ \\
\hline 1 & Expressway & 20 & 12 & 4000 & 0.5 & 40 & 20 \\
2 & Highway & 50 & 30 & 5400 & 0.7 & 20 & 40 \\
3 & Arterial & 40 & 40 & 4800 & 0.9 & 0 & 0 \\
\hline
\end{tabular}

\section{Multi-objective User Equilibrium}

In Sections 2.2 and 2.3, we have seen that equilibrium models considering the minimisation of generalised cost such as Equation (9) or travel time budget such as in Equation (11) do in fact consider the minimisation of the weighted sum of several objective functions, namely travel time $T_{k}(\mathbf{F})$ and monetary cost $M_{k}(\mathbf{F})$ in generalised cost user equilibrium (9), and expected travel time $E\left(T_{k}(\mathbf{F})\right)$ and standard deviation of travel time $\sigma_{T_{k}(\mathbf{F})}$ in travel time budget user equilibrium (11). As pointed out in Section 1, in our previous work (Wang \& Ehrgott 2013, Wang et al. 2014) we have shown that this model assumption of a linear combination of objective functions raises the possibility that 
some routes that are rational choices must necessarily have zero flow in an equilibrium solution. To overcome this limitation, we have studied bi-objective user equilibrium models regarding (toll) cost and travel time in Wang \& Ehrgott (2013) and expected value and standard deviation of travel time in Wang et al. (2014), respectively. In this paper, we extend our analysis to multi-objective equilibrium models considering expected value and standard deviation of travel time as well as monetary (toll) cost. In this section, we present general definitions and basic results on multi-objective network equilibrium models. For ease of notation, we assume one user class. Generalisations to multiple user classes are straightforward.

First, we repeat the definitions of efficient paths and (weak) multi-objective user equilibrium from Wang \& Ehrgott (2013). In the remainder of this section, let $G=(N, A)$ be a network, $Z \subset N \times N$ be a set of O-D pairs and for all $p \in Z$, let $D_{p}$ be the demand of O-D pair $p$. Let $C_{k}^{(i)}(\mathbf{F}), i=1, \ldots, r$ be $r$ cost functions of path $k$ and let $\mathbf{C}_{k}(\mathbf{F})$ denote the cost vector of path $k$. We assume that all cost functions $C_{k}^{(i)}(\mathbf{F}), i=1, \ldots, r$ are positive and continuous.

Definition 1 Let $\mathbf{F} \in \Omega$ be a feasible path flow vector.

1. Path $k$ is efficient, if there is no path $k^{\prime} \in K_{p}$ such that $C_{k^{\prime}}^{(i)}(\mathbf{F}) \leq C_{k}^{(i)}(\mathbf{F})$ for all $i=1, \ldots, r$ and $C_{k^{\prime}}^{(j)}(\mathbf{F})<C_{k}^{(j)}(\mathbf{F})$ for at least one $j \in\{1, \ldots, r\}$. If such a path $k^{\prime}$ exists then path $k^{\prime}$ dominates path $k$ and path cost vector $\mathbf{C}_{k^{\prime}}(\mathbf{F})$ dominates path cost vector $\mathbf{C}_{k}(\mathbf{F})$.

2. Path $k$ is weakly efficient, if there is no path $k^{\prime} \in K_{p}$ such that $C_{k^{\prime}}^{(i)}(\mathbf{F})<C_{k}^{(i)}(\mathbf{F})$ for all $i=1, \ldots, r$. If such a path $k^{\prime}$ exists then path $k^{\prime}$ strictly dominates path $k$ and path cost vector $\mathbf{C}_{k^{\prime}}(\mathbf{F})$ strictly dominates path cost vector $\mathbf{C}_{k}(\mathbf{F})$.

Definition 2 Let $\mathbf{F}^{*} \in \Omega$ be a feasible path flow vector.

1. Flow vector $\mathbf{F}^{*} \in \Omega$ is a multi-objective equilibrium flow, if whenever $\mathbf{C}_{k}\left(\mathbf{F}^{*}\right)$ dominates $\mathbf{C}_{k^{\prime}}\left(\mathbf{F}^{*}\right)$ for $k, k^{\prime} \in K_{p}$ for any $p \in Z$ then $F_{k^{\prime}}=0$.

2. Flow vector $\mathbf{F}^{*} \in \Omega$ is a weak multi-objective equilibrium flow, if whenever $\mathbf{C}_{k}\left(\mathbf{F}^{*}\right)$ strictly dominates $\mathbf{C}_{k^{\prime}}\left(\mathbf{F}^{*}\right)$ for $k, k^{\prime} \in K_{p}$ for any $p \in Z$ then $F_{k^{\prime}}=0$.

We present some basic general results concerning multi-objective equilibrium flows.

Proposition 1 A multi-objective equilibrium flow exists.

Proof:Because of the assumption of positive and continuous path cost functions, we know that the generalised path cost functions

$$
G_{k}(\mathbf{F}):=\sum_{i=1}^{r} C_{k}^{(i)}(\mathbf{F})
$$

are positive and continuous. Hence an equilibrium flow $\mathbf{F}^{*}$ with respect to $G_{k}$ exists. We show that this equilibrium flow $\mathbf{F}^{*}$ is a multi-objective equilibrium flow. 
Assume to the contrary that there is an O-D pair $p$ and two paths $k, k^{\prime} \in K_{p}$ with positive flow such that $\mathbf{C}_{k^{\prime}}\left(\mathbf{F}^{*}\right)$ dominates $\mathbf{C}_{k}\left(\mathbf{F}^{*}\right)$. Then $G_{k^{\prime}}\left(\mathbf{F}^{*}\right)<G_{k}\left(\mathbf{F}^{*}\right)$, contradicting the fact that $\mathbf{F}^{*}$ is an equilibrium flow with respect to $G_{k}$.

In user equilibrium models, the question of uniqueness of equilibrium flows is important. We shall see in Example 1 that in multi-objective user equilibrium, even if all path cost functions are strictly monotone, there are no uniqueness results. We, therefore, proceed to another general result on multiobjective user equilibrium. It states that equilibrium flows remain (at least weak) equilibrium flows as the number of objectives increases.

Theorem 1 Let $\emptyset \neq R \subset\{1, \ldots, r\}$ and let $\mathbf{F}^{*} \in \Omega$ be a multi-objective equilibrium flow with respect to the cost functions $C_{k}^{(i)}$ for $i \in R$. Then $\mathbf{F}^{*}$ is a weak multi-objective equilibrium flow with respect to cost functions $C_{k}^{(i)}, i=1, \ldots r$.

Proof:Assume to the contrary that $\mathbf{F}^{*}$ is not a weak multi-objective equilibrium flow. Then there exists some OD pair $p$ and two paths $k$ and $k^{\prime}$ in $K_{p}$ with positive flow such that $C_{k^{\prime}}^{(i)}\left(\mathbf{F}^{*}\right)<C_{k}^{(i)}\left(\mathbf{F}^{*}\right)$ for all $i=1, \ldots, r$. In particular, this implies that $C_{k^{\prime}}^{(i)}\left(\mathbf{F}^{*}\right)<C_{k}^{(i)}\left(\mathbf{F}^{*}\right)$ for all $i \in R$. Hence, in the equilibrium problem considering objectives $C_{k}^{(i)}$ for $i \in R$, cost vector $\mathbf{C}_{k}(\mathbf{F})$ strictly dominates cost vector $\mathbf{C}_{k^{\prime}}(\mathbf{F})$ and both $F_{k}^{*}>0$ and $F_{k^{\prime}}^{*}>0$, contradicting the property of multi-objective equilibrium flow for the equilibrium problem considering the objectives in $R$.

Because of the non-uniqueness of multi-objective user equilibrium flows special attention needs to be paid to ways of computing these flows. Theorem 2 provides a general method of scalarisation to compute multi-objective equilibrium flows.

Theorem 2 Let $s: \mathbb{R}^{r} \rightarrow \mathbb{R}$ be a strictly monotone and continuous function and let $\mathbf{F}^{*}$ be an equilibrium flow with respect to the path cost function $s\left(\mathbf{C}_{k}\left(\mathbf{F}^{*}\right)\right)$. Then $\mathbf{F}^{*}$ is a multi-objective equilibrium flow with respect to path cost functions $C_{k}^{(i)}\left(\mathbf{F}^{*}\right), i=1, \ldots, r$.

Proof:Let $\mathbf{F}^{*}$ be an equilibrium flow with respect to the path cost function $s\left(\mathbf{C}_{k}\left(\mathbf{F}^{*}\right)\right)$ and assume that it is not multi-objective equilibrium flow with respect to path cost functions $C_{k}^{(i)}\left(\mathbf{F}^{*}\right), i=$ $1, \ldots, r$. Then there exists some OD pair $p$ and two paths $k$ and $k^{\prime}$ in $K_{p}$ with positive flow such that $\mathbf{C}_{k^{\prime}}\left(\mathbf{F}^{*}\right)$ dominates $\mathbf{C}_{k}\left(\mathbf{F}^{*}\right)$. Because $s$ is a strictly decreasing function, this implies $s\left(\mathbf{C}_{k^{\prime}}\left(\mathbf{F}^{*}\right)\right)<$ $s\left(\mathbf{C}_{k}\left(\mathbf{F}^{*}\right)\right)$, contradicting the assumption.

In fact, the basic result of Theorem 2 shows that equilibrium models using generalised cost as in Equation (9), but also the nonlinear model of Larsson et al. (2002) using path cost function (10) compute bi-objective user equilibria with respect to the path cost functions $T_{k}(\mathbf{F})$ and $M_{k}(\mathbf{F})$.

Example 1 In the three-link example of Section 2.4, we will consider single-and multi-objective equilibrium flows with respect to the three cost functions toll $\tau_{k}:=\tau_{a}^{1}$, expected travel time $E\left(T_{k}\right)$ and standard deviation of travel time $\sigma_{t_{k}}$ as defined in (15) and (16). All equilibrium flows are illustrated in Figure 2, which shows $\Omega$ represented in $\mathbb{R}^{2}$ by values of $f_{1}$ and $f_{2}$. 


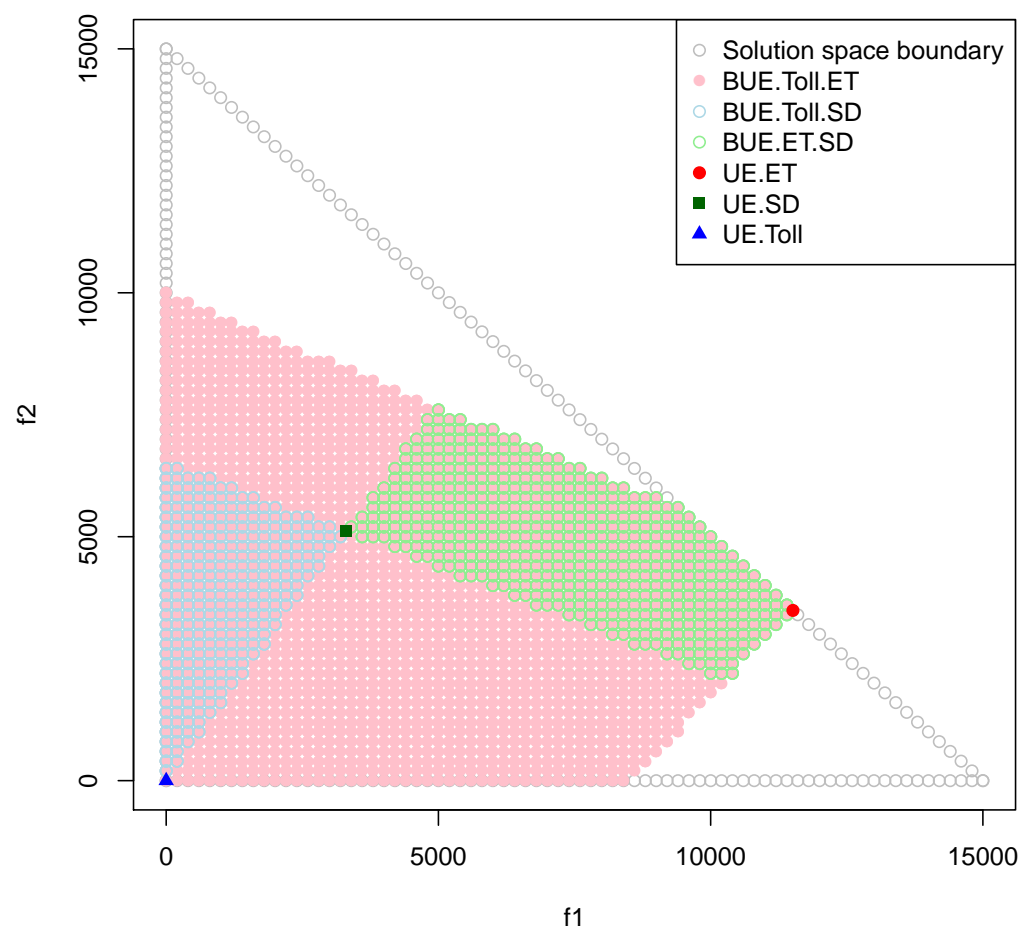

Figure 2: Equilibrium flows with one, two and three objectives for $\tau_{1}=40, \tau_{2}=20, \tau_{3}=0$. 


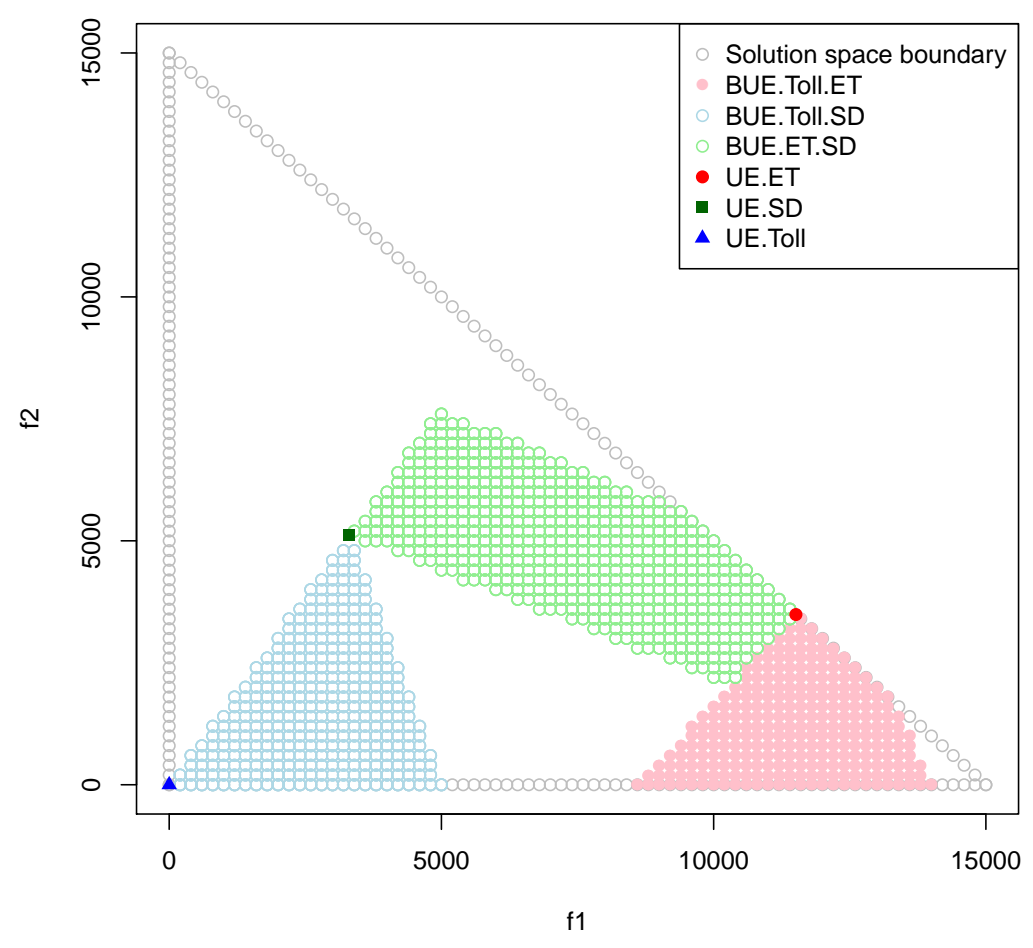

Figure 3: Equilibrium flows with one, two and three objectives for $\tau_{1}=20, \tau_{2}=40, \tau_{3}=0$.

The (unique) single-objective equilibrium flows are shown by the blue triangle (toll, UE.Toll), green square (standard deviation of travel time, UE.SD), and red circle (expected travel time, UE.ET), respectively. All three combinations of two objectives result in regions of bi-objective user equilibrium flows, indicated by pale red (BUE.TollET), green (BUE.TollSD) and blue (BUE.ETSD) circles. Finally, in this particular example, the region of three-objective user equilibrium flows coincides with the biobjective toll versus expected travel time user equilibrium flows. Because toll ranks the three routes in the opposite way of expected travel time, addition of the third objective does not add any further equilibrium flows. The inclusions stated in Theorem 1 are clearly verified. Notice that the inclusions postulated may or may not be strict.

In a second example, we change the tolls to $\tau_{a}^{2}$, so that $\tau_{1}=20, \tau_{2}=40, \tau_{3}=0$. Here, the toll for the faster but less reliable expressway is lower than the toll for the slower but more reliable highway. This affects the regions of bi-objective and three-objective user equilibrium flows. In Figure 3, the three-objective user equilibrium flows form the convex hull of all the bi-objective user equilibrium regions. 


\section{The Time Surplus Maximisation Model}

In previous work (Wang \& Ehrgott 2013, Wang et al. 2014) we have investigated bi-objective user equilibrium models considering the path cost functions $T_{k}(\mathbf{F}), \tau_{k}(\mathbf{F}), E\left(T_{k}(\mathbf{F})\right.$, and $\sigma_{T_{k}}(\mathbf{F})$. Specifically, in Wang \& Ehrgott (2013), we introduced the time surplus maximisation equilibrium model as a new model for route choice behaviour in tolled road networks considering the two objective functions travel time $C_{k}^{(1)}(\mathbf{F})=T_{k}(\mathbf{f})=\sum_{a \in k} t_{a}\left(f_{a}\right)$, where $t_{a}\left(f_{a}\right)$ is the travel time function (7) and toll $C_{k}^{(2)}(\mathbf{F})=M_{k}(\mathbf{f})=\tau_{k}=\sum_{a \in k} \tau_{a}$, with exogenously defined link tolls $\tau_{a}$.

For every O-D pair $p \in Z$, users of class $m$ have an indifference function between toll and time, a function $T_{p m}^{\max }: \mathbb{R} \rightarrow \mathbb{R}$ that is strictly decreasing, i.e. $T_{p m}^{\max }\left(\tau_{k}^{1}\right)<T_{p m}^{\max }\left(\tau_{k}^{2}\right)$ if $\tau_{k}^{1}>\tau_{k}^{2}$, where $T_{p m}^{\max }(\tau)$ is interpreted as the maximum time they would be willing to spend to travel from the origin to the destination of O-D pair $p$ if the toll is $\tau$. The strict decreasing property of $T_{p m}^{\max }$ takes into account that users would expect to spend less time in traffic if they need to pay a higher toll.

Time surplus is defined as the time that the user would be willing to spend minus the actual travel time, i.e. given the indifference curves $T_{p m}^{\max }$ for all $p \in Z$ and $m \in M$, time surplus for path $k \in K_{p}$ and user class $m$ is

$$
T S_{k m}(\mathbf{F}):=T_{p m}^{\max }\left(\tau_{k}\right)-T_{k}(\mathbf{f})=T_{p m}^{\max }\left(\sum_{a \in k} \tau_{a}\right)-\sum_{a \in k} t_{a}\left(f_{a}\right) .
$$

Assuming that users choose the path $k^{*}$ with maximum time surplus, i.e.

$$
k^{*}(m)=\operatorname{argmax}\left\{T S_{k m}(\mathbf{F}): k \in K_{p}\right\}
$$

we obtain the time surplus maximisation (bi-objective) user equilibrium (TSmaxBUE) condition by using $\Delta_{p m}-T S_{k m}(\mathbf{F})$ for $C_{k m}(\mathbf{F})$ in the user equilibrium definition of Equations (1) - (5), where $\Delta_{p m}$ is a sufficiently large constant, e.g. $\Delta_{p m}=\max _{k \in K_{p}} T_{k}\left(D_{p}\right)$, to ensure positivity of $T_{k m}(\mathbf{F})$. Notice that it is natural to assume that $T_{p m}^{\max }$ is bounded by $\max _{k \in K_{p}} T_{k}\left(D_{p}\right)$, the longest travel time if all demand for O-D pair $p$ is on a single path.

In Wang \& Ehrgott (2013) we have shown that time surplus maximisation bi-objective user equilibrium characterises all bi-objective user equilibrium flows with respect to the objectives travel time and toll, which we state as Theorem 3. Moreover, in Wang \& Ehrgott (2013) we show that generalised cost user equilibrium with objective function (9) is a special case of time surplus maximisation bi-objective user equilibrium. Note that this result also follows from Theorem 2 in Section 3.

Theorem $3 \mathbf{F}^{*}$ is a bi-objective equilibrium flow, with respect to objectives $C_{k}^{(1)}(\mathbf{F})=T_{k}(\mathbf{F})$ and $C_{k}^{(2)}(\mathbf{F})=\tau_{k}$ if and only if there exist indifference functions $T_{p m}^{\max }$ for all $p \in Z$ and $m \in M$ such that $\mathbf{F}^{*}$ is also a TSmaxBUE flow.

In this paper, we are interested in a single-objective user equilibrium model that allows to compute three objective user equilibrium flows. In Section 5 we proceed to extend the TSmaxBUE concept to the case of travel time uncertainty using the concept of travel time budget. 


\section{Time Surplus Maximisation under Travel Time Uncertainty}

Let us consider how to apply the time surplus concept to model individual route choices with different views towards travel time reliability.

We first need to introduce variability of travel time in the time surplus function defined in Wang \& Ehrgott (2013) as in (19). Here we apply the concept of travel time budget in Lo et al.'s model. We define $\rho$ as the desired level of reliability, or equivalently, $1-\rho$ as the critical level of risk. $\rho$ equals the desired percentage of times that an individual can get to the destination within the allotted travel time. A risk neutral user will have a $\rho$-value of $50 \%$ while a risk averse user might have a $\rho$-value of $95 \%$. As in Wang et al. (2014), we assume that users are all either risk neutral or risk averse, i.e. $\rho \in[0.5,1)$.

Now we define travel time budget surplus as in Equation (21),

$$
T B S_{k m}(\mathbf{F})=T_{p m}^{\max }\left(\tau_{k}\right)-T_{k m}^{\rho}(\mathbf{F})
$$

where

$$
P\left(T_{k} \leq T_{k m}^{\rho}\right)=\rho .
$$

For risk neutral users, $T_{k m}^{\rho}$ will be the median value of the travel time on path $k$, while for risk averse users, $T_{k m}^{\rho}$ will be higher than the median, depending on the level of risk aversion $\rho$.

Now let us consider a risk neutral user with a convex indifference curve as shown in Figure 4. The time budget surplus of this user can be determined by comparing the indifference curve with the median travel time curve. In this case, Route 1 will be the optimal choice for this individual as this route has the highest time budget surplus. On the other hand, a risk averse user with the same indifference curve and $\rho=95 \%$, as shown in Figure 5, will consider Route 2 an optimal choice, as this route provides the least negative time budget surplus.

Similarly, we can consider users with a different attitude towards risk, but with a concave indifference curve as shown in Figures 6 and 7. Again, their choices would be different even though they have the same indifference curve. A risk neutral user will find Route 1, with the largest positive time budget surplus, the most attractive, while a risk averse user will choose Route 2 instead as it has the least negative time budget surplus value.

As discussed in Section 2.3, we know that we can guarantee the travel time reliability stated in Equation (22), if we set $T_{k m}^{\rho}$ equal to the time budget $E\left(T_{k}\right)+\lambda_{m} \sigma_{T_{k}}$, as presented in Equations (17) and (18). Therefore, to model user equilibrium considering toll, travel time, and travel time reliability we propose a user equilibrium model considering the path benefit function

$$
T B S_{k m}(\mathbf{F})=T_{p m}^{\max }\left(\tau_{k}\right)-E\left(T_{k}\right)-\lambda_{m} \sigma_{T_{k}}
$$

Notice that Equation (23) is obtained from Equation (21) using the time budget formula of Equation (11). We consider multiple user classes $m \in M$, where each user class $m$ has its specific indifference curve $T_{p m}^{\max }\left(\tau_{k}\right)$ and attitude towards risk, quantified in parameter $\rho_{m}$ and the corresponding 


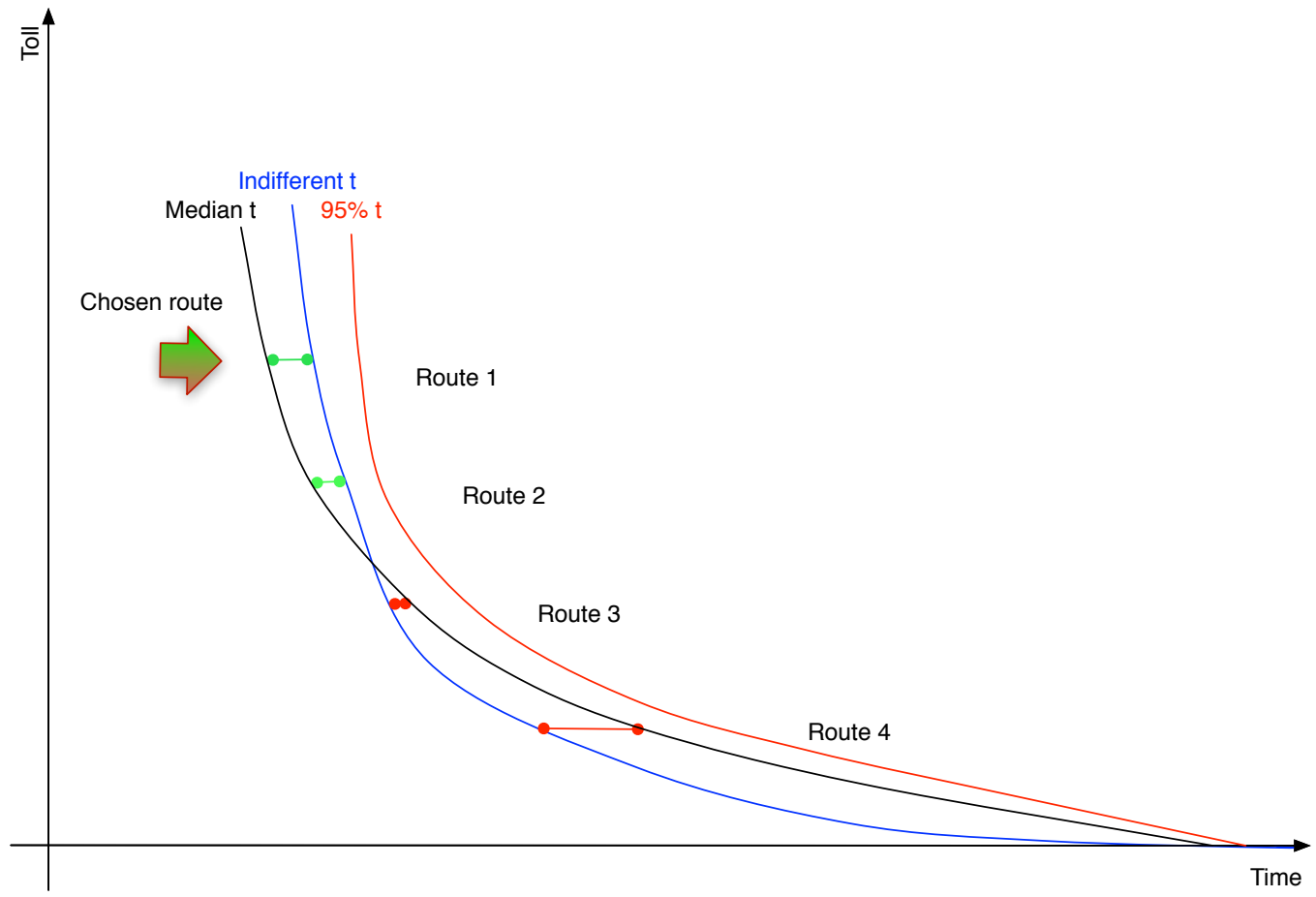

Figure 4: Travel time budget surplus for risk neutral users with convex indifference curve.

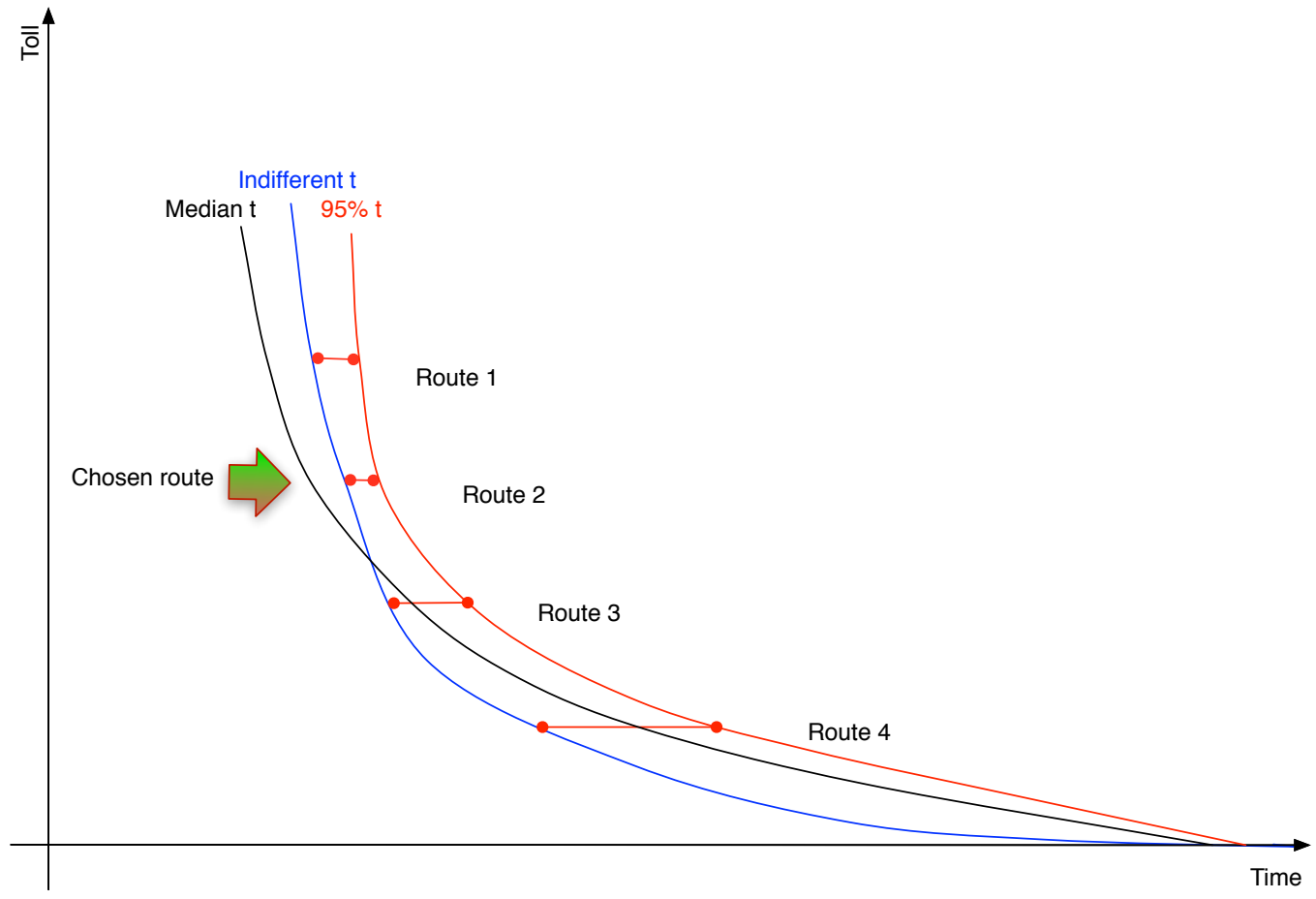

Figure 5: RTravel time budget surplus for risk averse users with convex indifference curve. 


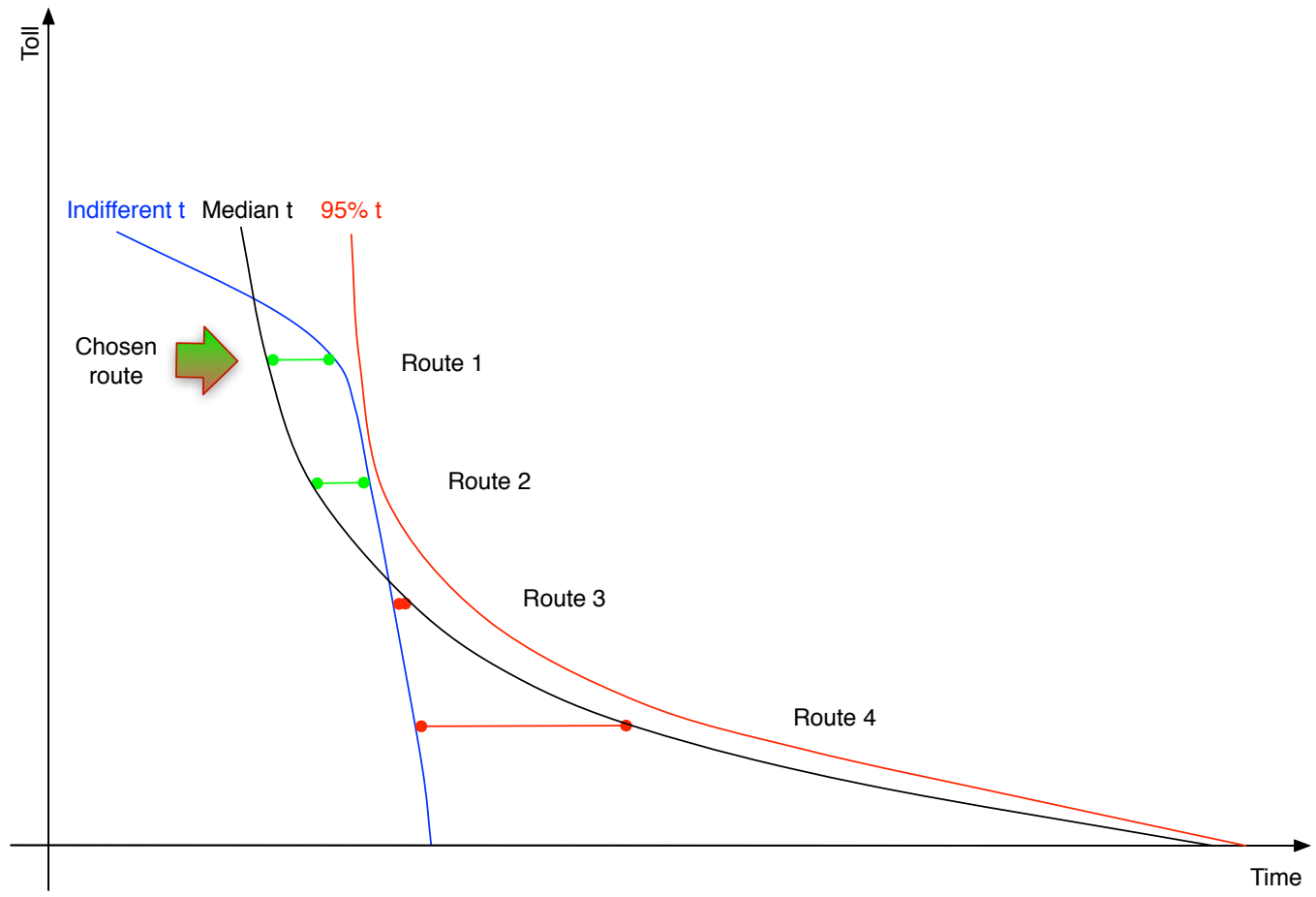

Figure 6: Travel time budget surplus for risk neutral users with concave indifference curve.

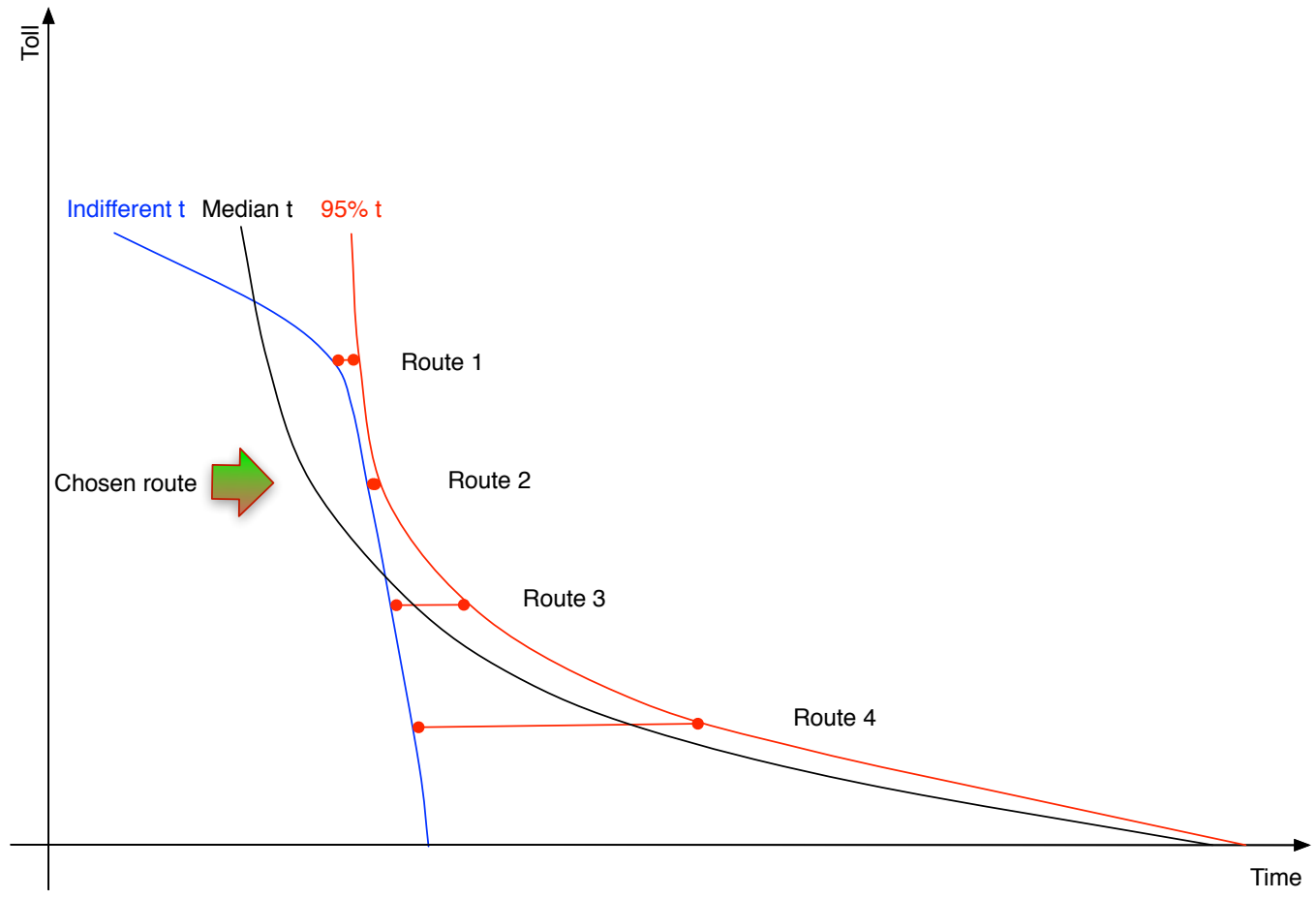

Figure 7: Travel time budget surplus for risk averse users with concave indifference curve. 
value $\lambda_{m}$. Hence, our multi-user class equilibrium model incorporating users' trade-offs between travel time and toll as well as attitude towards risk (measured by standard deviation of travel time) is based on the class-specific path benefit function (23), which is turned into a cost function by subtracting it from a sufficiently large constant such as $\Delta_{p m}$ as in Section 4, but considering the least capacity $\rho_{a} C_{a}$ in its definition. We call this model the Time Budget Surplus Maximisation (Three-Objective) User Equilibrium (TBSmaxTUE) model. We call a feasible flow vector $\mathbf{F}^{*} \in \Omega$ that satisfies the (multi-user) class equilibrium conditions with path cost function $\Delta_{p m}-T B S_{k m}(\mathbf{F})$ a TBSmaxTUE flow. The name is justified by Theorems 4 and 5 below, which show that a TSBmaxTUE equilibrium flow is indeed a three-objective use equilibrium flow with respect to the three constituent objectives.

Replicating the result of Theorem 3 we can immediately state the equivalence between time budget surplus user equilibrium and bi-objective user equilibrium considering path cost functions $\tau_{k}$ (toll) and $B_{k m}$ (travel time budget).

Theorem 4 Path flow vector $\mathbf{F}^{*} \in \Omega$ is a bi-objective equilibrium flow with respect to objectives $C_{k m}^{(1)}(\mathbf{F})=B_{k m}(\mathbf{F})$ and $C_{k m}^{(2)}(\mathbf{F})=\tau_{k}$ if and only if there exist indifference functions $T_{p m}^{\text {max }}$ such that $\mathbf{F}^{*}$ is also a TBSmaxTUE flow.

We will further link travel time budget surplus equilibrium with the general framework of multiobjective user equilibrium discussed in Section 3. That is, we consider in particular three-objective user equilibrium with cost functions toll, expected travel time, and standard deviation of travel time.

Theorem 5 Let $\mathbf{F}^{*} \in \Omega$ be a bi-objective equilibrium flow with respect to path cost functions $C_{k m}^{(1)}(\mathbf{F})$ $=E\left(T_{k}(\mathbf{F})\right)+\lambda_{m} \sigma_{T_{k}(\mathbf{F})}$ and $C_{k m}^{(2)}(\mathbf{F})=\tau_{k}$. Then $\mathbf{F}^{*}$ is also a three-objective user equilibrium flow with respect to toll, expected travel time, and standard deviation of travel time.

Proof:Assuming the contrary, there would be an OD pair $p$, a user class $m$ and two paths $k, k^{\prime} \in K_{p}$ with positive flow such that

$$
\begin{aligned}
E\left(T_{k}\right) & \leq E\left(T_{k^{\prime}}\right) \\
\sigma_{T_{k}} & \leq \sigma_{T_{k}^{\prime}} \\
\tau_{k} & \leq \tau_{k^{\prime}}
\end{aligned}
$$

with at least one of the inequalities being strict. If the strict inequality occurs in the expected travel time or standard deviation component, then we have $E\left(T_{k}\right)+\lambda \sigma_{T_{k}}<E\left(T_{k^{\prime}}\right)+\lambda \sigma_{T_{k^{\prime}}}$ (notice that $\lambda>0$ ). Together with $\tau_{k} \leq \tau_{k^{\prime}}$, the cost vector of path $k$ dominates the cost vector of path $k^{\prime}$ when considering toll and time budget, contradicting the property of multi-objective equilibrium flow of $\mathbf{F}^{*}$ with respect to time budget and toll.

The case of strict inequality with toll is completely analogous.

Example 2 Once again, we illustrate the results of Theorems 4 and 5 using the three-link example of Section 2.4. Here we consider only a single user class and $\rho=0.9$. Figures 8 and 9 show the 


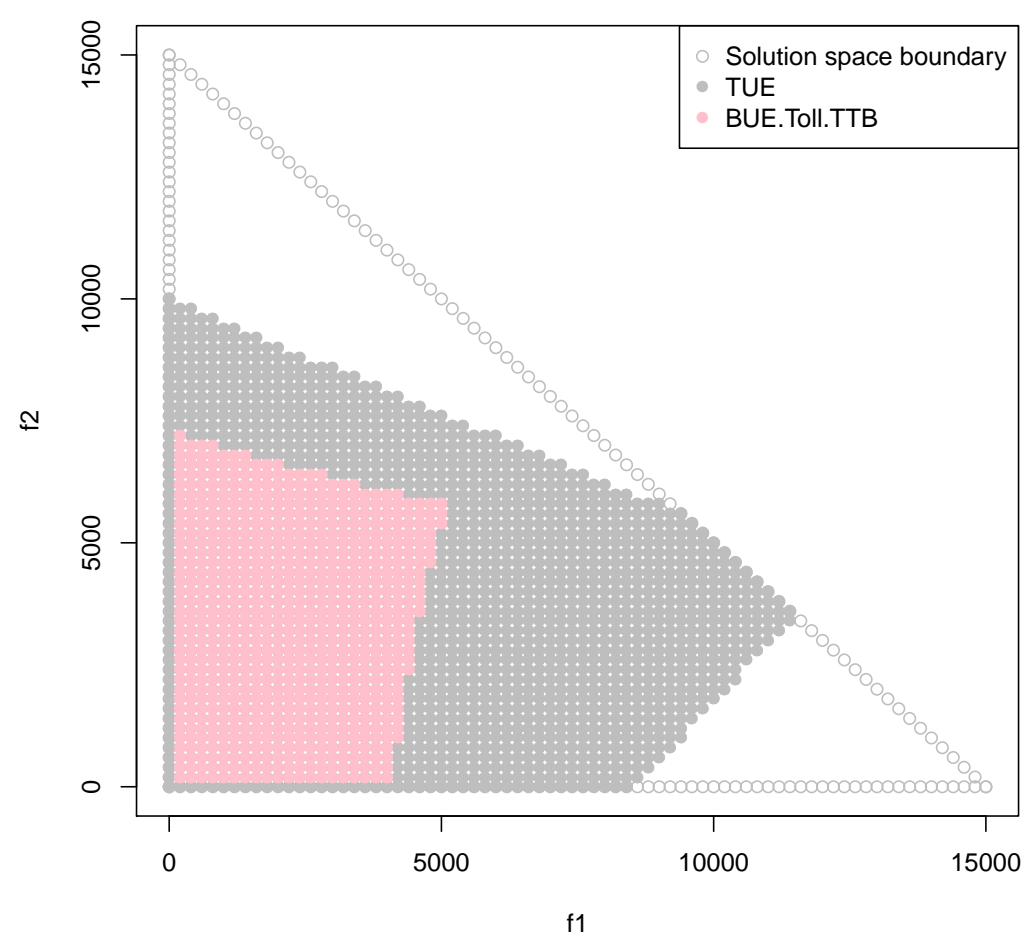

Figure 8: Equilibrium flows for toll versus travel time budget and three objectives, $\tau_{1}=40, \tau_{2}=$ $20, \tau_{3}=0$.

region of three-objective user equilibrium (grey, TUE) and bi-objective user equilibrium for toll versus travel time budget (pale red, BUE.TollTTB) for the cases of toll as in Table 1 (Figure 8) and for $\tau_{1}=20, \tau_{2}=40, \tau_{3}=0$ (Figure 9), see also Example 1.

Figures 8 and 9 clearly show that for fixed $\rho$ and indifference curve, the bi-objective equilibrium flows are (proper) subsets of three-objective equilibrium flows. The reverse, whether for any threeobjective user equilibrium flow $\mathbf{F}^{*}$ there exists an indifference curve and $\rho$ such that $\mathbf{F}^{*}$ is a biobjective user equilibrium with cost functions toll and travel time budget, remains an open question for now.

\subsection{An NCP Formulation of the Time Budget Surplus Maximisation Model}

Recall that the time budget surplus function $T B S_{k m}(\mathbf{F})$ defined in Equation (23) is strictly increasing, continuous and positive. Therefore, an equilibrium flow with respect to $T B S_{k m}(\mathbf{F})$ can be computed by minimising a gap function related to the nonlinear complementarity formulation associated with the equilibrium problem. We use the function $\Phi(a, b)=\sqrt{a^{2}+b^{2}}-(a+b)$ that was also used by Lo \& Chen (2000). Let 


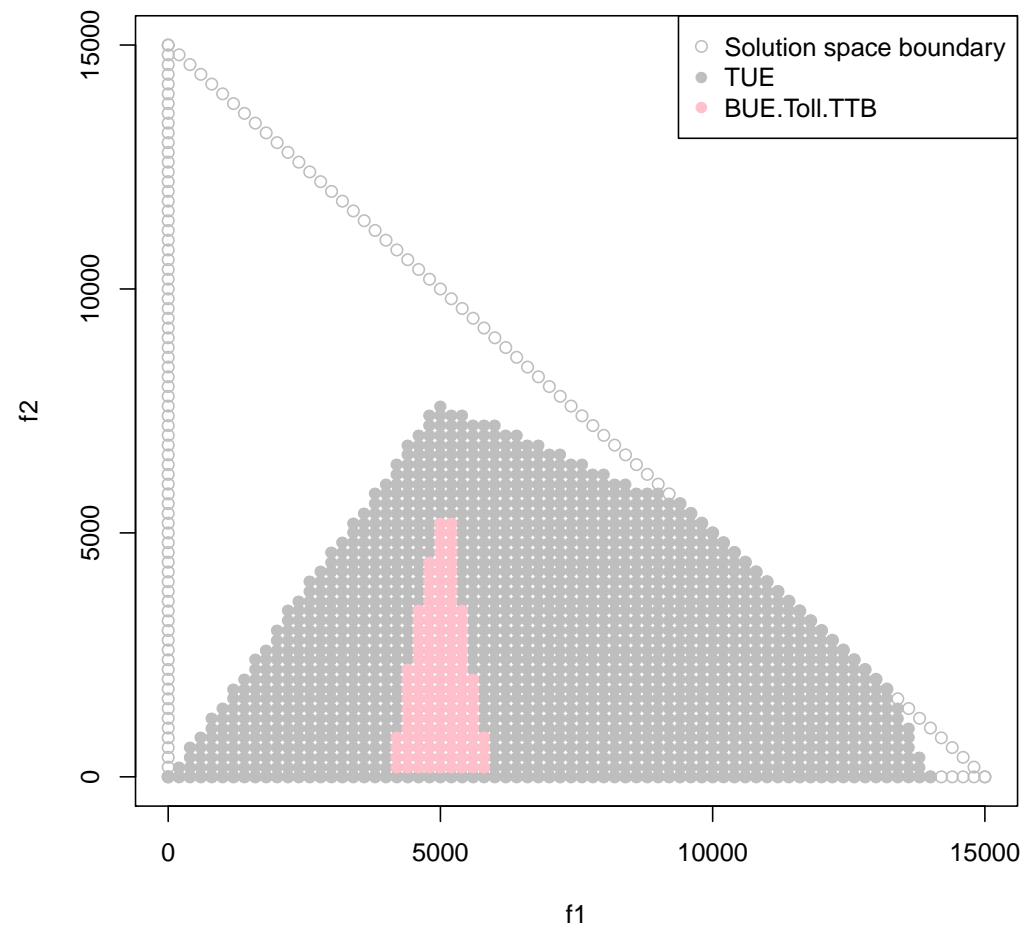

Figure 9: Equilibrium flows for toll versus travel time budget and three objectives, $\tau_{1}=20, \tau_{2}=$ $40, \tau_{3}=0$. 
- user class $m$ be defined by a specific indifference curve $T_{p m}^{\max }$ and risk attitude $\rho_{m}$;

- $D_{p m}$ be the demand for OD pair $p$ and user class $m$;

- $f_{k p m}$ be the flow of user class $m$ on path $k$ for OD pair $p$;

- $\pi_{p m}$ be a variable for the minimal path cost of user class $m$ for OD pair $p$; and

- $\eta_{k p m}:=\Delta_{k m}-T B S_{k m}$ be the "cost" of path $k \in K_{p}$ for user class $m$, where $\Delta_{k m}$ is a sufficiently large number.

Then the gap function $G(\mathbf{F})$ defined by $\Phi$ is

$$
G(\mathbf{F}):=\sum_{m \in M} \sum_{p \in Z} \sum_{k \in K_{p}} \frac{1}{2} \Phi^{2}\left(f_{k p m}, \eta_{k p m}-\pi_{p m}\right)+\sum_{m \in M} \sum_{p \in Z} \frac{1}{2} \Phi^{2}\left(\pi_{p m}, \sum_{k \in K_{p}}-D_{p m}\right)
$$

and it holds that $\mathbf{F}$ is an equilibrium flow if and only if $G(\mathbf{F})=0$. The variables in the NCP model are the path flows for each OD pair, path, and user class as well as the minimum path costs for each OD pair and user class.

To summarise our results, we have suggested a three-objective user equilibrium model as a general framework to model the influence of the three most important factors influencing route choice behaviour in tolled road networks, namely expected travel time, travel time reliability, and monetary cost. Because such a multi-objective equilibrium model has in general infinitely many equilibrium flows, we have proposed a time surplus maximisation model under uncertainty to operationalise the concept, i.e. to compute three objective user equilibrium solutions. This model combines the timesurplus maximisation equilibrium for route choice modelling in tolled road networks (Wang \& Ehrgott 2013) with the time budget concept to model travel time reliability (Lo et al. 2006).

\section{Numerical Example}

In this section, we apply the travel time budget surplus maximisation model to the three-link example of Section 2.4. Since there is only one OD pair, we omit the index $p$ hereafter. We consider six user classes altogether. They are characterised by three indifference curves $T_{1}^{\max }, T_{2}^{\max }$ and $T_{3}^{\max }$ as shown in Table 2. For each indifference curve, we have two user classes, one representing risk neutral users with $\rho_{m}=0.50$, and another representing risk averse users with $\rho_{m}=0.95$. The tolls are as specified for $\tau_{a}^{1}$ in Table 1 and routes are identified by their toll in figures below. The problem has been solved in MATLAB using the NCP formulation described in Section 5.1. The tolls are as specified for $\tau_{a}^{1}$ in Table 1 and routes are identified by their toll in figures.

\subsection{Equilibrium Flows}

The equilibrium flows for the six different user classes are shown in Figure 10. All risk neutral users

will travel on the toll-free route. For risk averse users, those with lower maximum time willing to 
Table 2: Maximum time willing to spend.

\begin{tabular}{lrrr}
\hline Route & \multicolumn{3}{c}{ Indifference curve } \\
$k$ & $T_{1}^{\text {max }}$ & $T_{2}^{\text {max }}$ & $T_{3}^{\text {max }}$ \\
\hline 1 & 12.5 & 17.5 & 22.5 \\
2 & 32.5 & 37.5 & 42.5 \\
3 & 65.0 & 75.0 & 85.0 \\
\hline
\end{tabular}

spend (indifference curves $T_{1}^{\max }$ and $T_{2}^{\max }$ ) will choose to pay, while all risk averse users with the highest maximum time willing to spend (indifference curve $T_{3}^{\max }$ ) will travel on the toll-free route. This is consistent with our expectations that it would be those who are risk averse and more willing to pay who would travel on the tolled (and more reliable) routes.

\subsection{TBSmaxTUE Conditions}

To check that the TBSmaxBUE conditions are satisfied, the time budget surplus values for all six user classes are compared at equilibrium flows. For risk neutral users, Figure 11 shows that the toll-free route has the highest time budget surplus for users with all three indifference curves and thus is the only route with positive flow. For risk averse users, on the other hand, Figure 12 shows that the tolled routes for users with indifference curves $T_{1}^{\max }$ and $T_{2}^{\max }$ have the least negative time budget surplus and thus have positive flows. The toll-free route has the highest positive time budget surplus for users with indifference curve $T_{3}^{\max }$ and thus is the only route with positive flow. Note that as expected the time budgets for risk averse users are higher than those for the risk neutral ones.

\section{Conclusion and Outlook}

In this paper, we propose a novel approach to incorporate the consideration of the three most important factors influencing route choice behaviour in a road network, namely, travel time, travel time reliability and monetary cost. Our proposed model is based on the concept of three-objective user equilibrium, assuming that all users aim to (1) minimise expected travel time; (2) minimise travel time variability; and (3) minimise toll (monetary cost).

We further develop the TSmaxBUE model introduced in Wang \& Ehrgott (2013), where we introduced the concept of time surplus maximisation to model user preferences in a general form rather than using a VOT value. We assume that for any given OD pair and a specific toll value, there is a limit on the time that an individual would be willing to spend (forming an indifference curve between toll and time for the individual). Each individual can have his/her own preference represented by this indifference curve between toll and time. Time surplus is defined as the time that an individual is willing to spend minus the actual travel time. Given a set of routes, the one with the highest (or least 


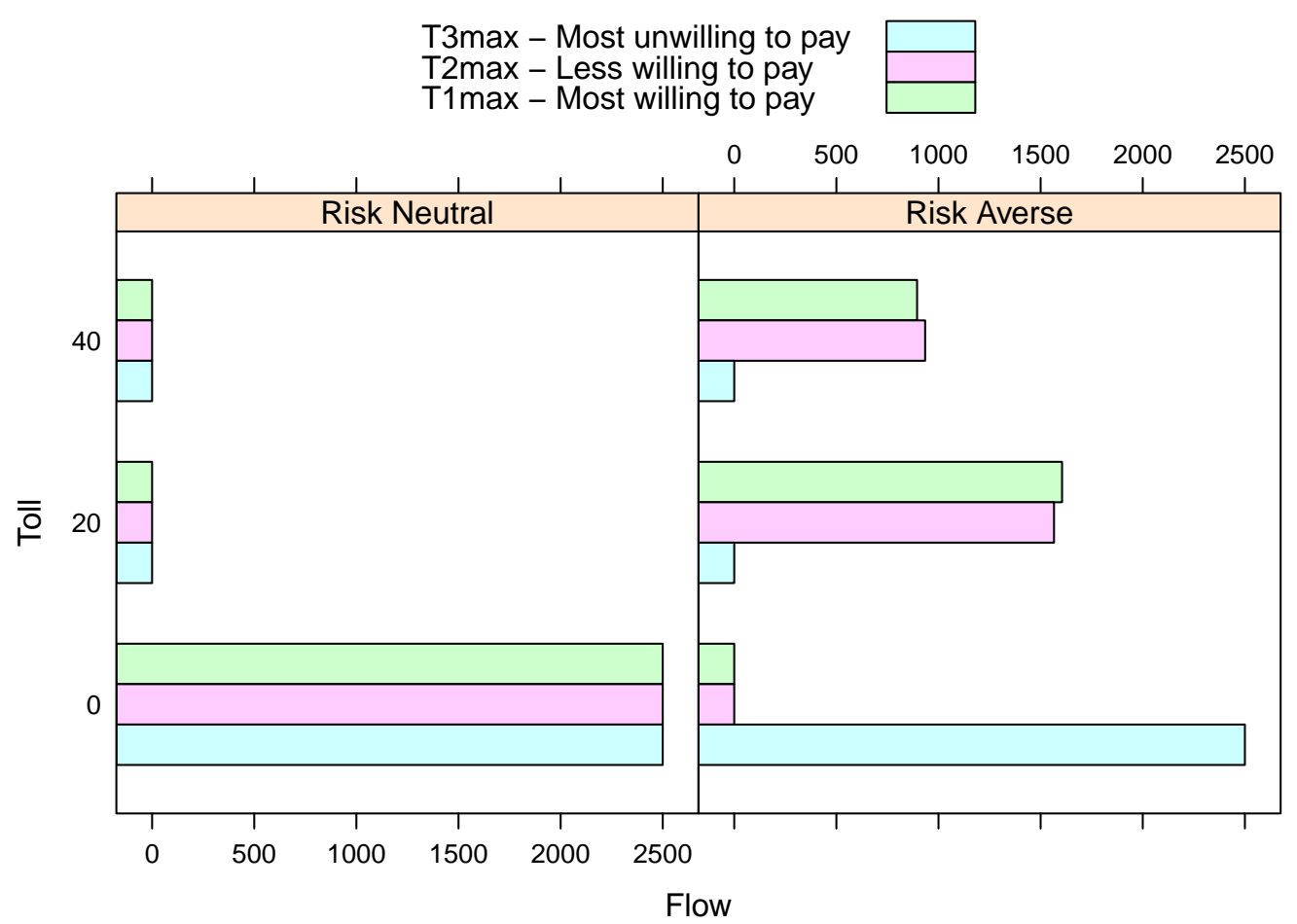

Figure 10: Equilibrium flows. 


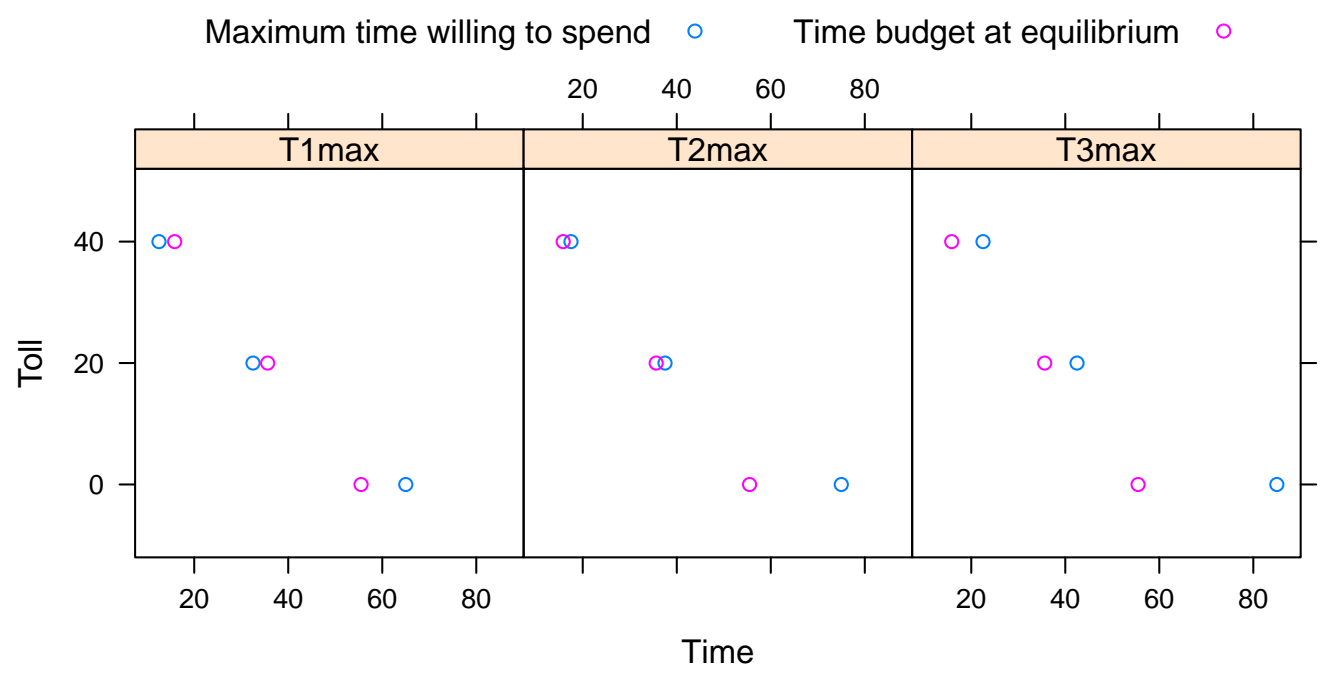

Figure 11: Time budget surplus of risk neutral users. 


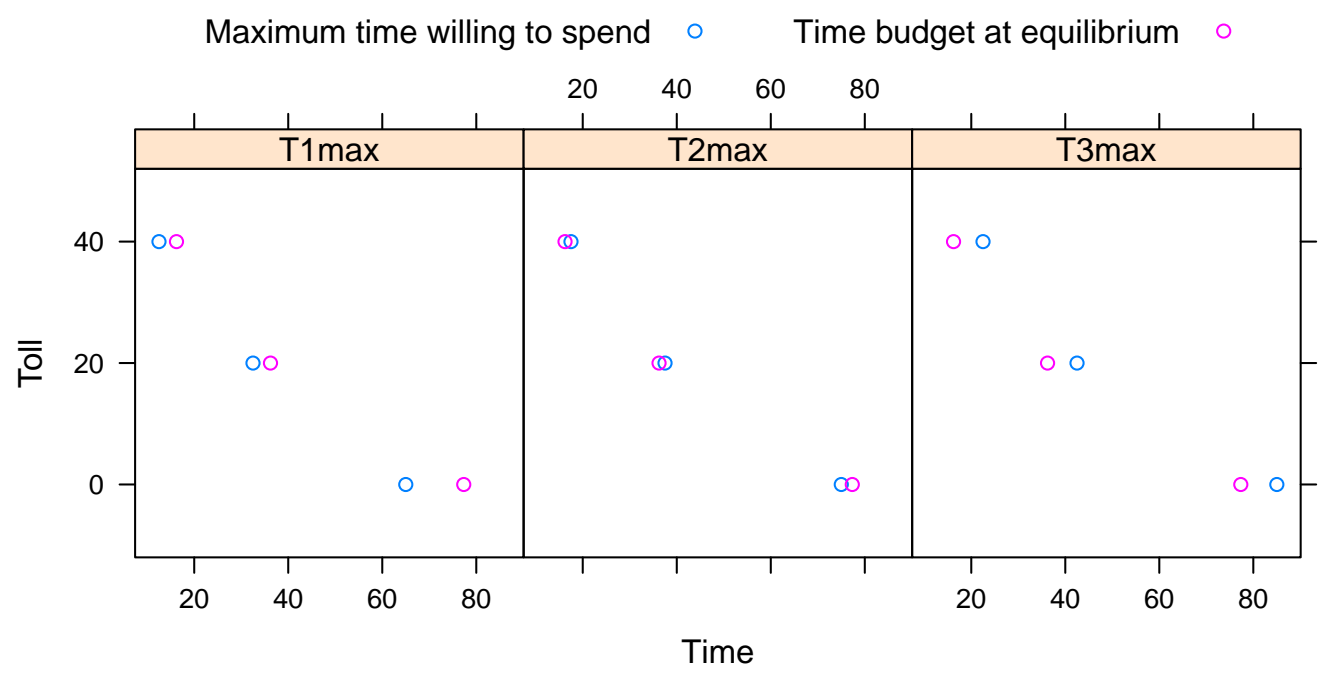

Figure 12: Time budget surplus of risk averse users. 
negative) time surplus will be the preferred route for the individual.

We then incorporate the concept of travel time budget (Lo et al. 2006) into the time surplus maximisation concept. For a given toll, a user will have two limits, one on the maximum travel time a user is willing to spend, and one on the minimum level of travel time reliability. Here, travel time reliability is defined as the probability of arriving at the destination within the allocated travel time budget. Based on the desired level of reliability, one can determine the travel time budget required on a particular route that will satisfy this level of reliability. Whether this route is attractive or not to an individual would depend on how much this required travel time budget differs from the maximum time the user is willing to spend given a particular toll. We define time budget surplus as the maximum time a user is willing to spend minus the travel time budget required for a desired level of travel time reliability, which is effectively a measure of the level of attractiveness. The route with the highest (or least negative) time budget surplus would be the most attractive option. We assume that each individual can be characterised by (1) an indifference curve between toll and maximum time willing to spend; and (2) the desired minimum level of reliability. In this way, we can determine the choice of each individual based on his/her own preference, modelled by the two characteristics. This becomes our proposed TBSmaxTUE model.

This concept can also be applied in a toll-free road network. As mentioned earlier, Abdel-Aty et al. (1995) identified that the third important factor affecting route choice is distance, which can be considered as a proxy variable of vehicle operating cost. One would expect that users will not choose a longer route unless the travel time on this route is expected to be shorter or the travel time reliability is higher. By replacing toll with distance, we can characterise each individual with (1) an indifference curve between distance and maximum time willing to spend for the desired travel time reliability; and (2) the desired minimum level of reliability.

We can further represent user groups by identifying a set of toll-time or distance-time indifference curves and their desired minimum level of reliability within each group represented by each curve. Each user class is identified by the combination of the two characteristics. We can then deduce the time budget surplus maximisation user equilibrium condition, whereby all individuals are travelling on the route with the highest time budget surplus value among all the efficient routes (based on the trade off between toll and time or distance and time) between each O-D pair.

Further research topics include consideration of elastic demand and other factors that might have strong influence on route choice behaviour. For example, users might not see the use of credits in emission trading schemes as a monetary cost. It is worthwhile to look into the possibility of modelling the effect of credits not as part of a multi-objective path cost function.

\section{Acknowledgments}

This research was partially supported by the Marsden Fund, grant number 9075362506. 


\section{References}

Abdel-Aty, M. A., Kitamura, R., \& Jovanis, P. P. 1995. Investigating effect of travel time variability on route choice using repeated-measurement stated preference data. Transportation Research Record, 1493, 3945 .

Bar-Gera, H. 2010. Traffic assignment by paired alternative segments. Transportation Research Part B, 44(8-9), 1022-46.

Brownstone, D., \& Small, K.A. 2005. Valuing time and reliability: Assessing the evidence from road pricing demonstrations. Transportation Research Part A, 39, 279-293.

Bureau of Public Roads. 1964. Traffic Assignment Manual. U.S. Department of Commerce, Urban Planning Division, Washington D.C.

Carrion, Carlos, \& Levinson, David. 2012. Value of travel time reliability: A review of current evidence. Transportation Research Part A: Policy and Practice, 46(4), 720 - 741.

Chen, A., Oh, J.S., Park, D., \& Recker, W. 2010. Solving the bicriteria traffic equilibrium problem with variable demand and nonlinear path costs. Applied Mathematics and Computation, 217, 3020-3031.

Dafermos, S., \& Sparrow, F. 1969. The traffic assignment problem for a general network. Journal of Research of the National Bureau of Standards B, 73 B, 91-118.

Dial, R.B. 1996. Bicriterion Traffic Assignment: Basic Theory and Elementary Algorithms. Transportation Science, 30(2), 93-111.

Florian, M. 2006. Network Equilibrium Models for Analyzing Toll Highways. Pages 105-115 of: Lawphongpanich, S., Hearn, D. W., \& Smith, M. J. (eds), Mathematical and Computational Models for Congestion Charging. Springer, New York.

Florian, M., \& Hearn, D. 1995. Network Equlibrium Models and Algorithms. Pages 485-550 of: Ball, M.O. (ed), Handbooks in Operations Research and Management Science, vol. 8.

Florian, Michael, Constantin, Isabelle, \& Florian, Dan. 2009. A New Look at Projected Gradient Method for Equilibrium Assignment. Transportation Research Record, 2090, 10-16.

Frank, F., \& Wolfe, P. 1956. An algorithm for quadratic programming. Naval Research Logistics Quarterly, 3, 95-110.

Gabriel, S.A., \& Bernstein, D. 1997. The traffic equilibrium problem with nonadditive path costs. Transportation Science, 31, 337-348.

Jackson, W.Burke, \& Jucker, James V. 1982. Empirical study of travel time variability and travel choice behaviour. Transportation Science, 16(4), 460-475. cited By (since 1996) 32.

Jayakrishnan, A., Tsai, W. K., \& Rajadhyaksha, S. 1994. A faster path-based algorithm for traffc assignment. Transportation Research Record, 1443, 75-83.

Lam, T.C., \& Small, K.A. 2001. The value of time and reliability: Measurement from a value pricing experiment. Transportation Research Part E, 37, 231-251.

Larsson, T., Lindberg, P. O., Patriksson, M., \& Rydergren, C. 2002. On Traffic Equilibrium Models with a Nonlinear Time/Money Relation. Chap. 2, pages 19-31 of: Patriksson, M., \& Labbé, M. (eds), Transportation Planning. Kluwer Academic Publishers, Secaucus.

Leurent, F. 1993. Cost versus time equilibrium over a network. European Journal of Operational Research, 71, $205-221$. 
Li, Z., Hensher, D. A., \& Rose, J. M. 2010. Willingness to pay for travel time reliability in passenger transport: A review and some new empirical evidence. Transportation Research Part E, 46, 384 - 403.

Liu, H., Recker, W., \& Chen, A. 2004. Uncovering the contribution of travel time reliability to dynamic route choice using real-time loop data. Transportation Research Part A, 38, 435-453.

Lo, H. K., \& Chen, A. 2000. Traffic equilibrium problem with route-specific costs: Formulation and algorithms. Transportation Research Part B, 34, 493-513.

Lo, H. K.., \& Tung, Y.-K. 2003. Network with degradable links: Capacity analysis and design. Transportation Research Part B, 37, 345 - 363.

Lo, H. K., Luo, X. W., \& Siu, B. W. Y. 2006. Degradable transport network: travel time budget of travellers with heterogeneous risk aversion. Transportation Research Part B, 40, 792-806.

Perederieieva, O., Ehrgott, M., Raith, A., \& Wang, J.Y.T. 2015. A framework for and empirical study of algorithms for traffic assignment. Computers \& Operations Research, 54, 90-107.

Small, K.A. 1982. The scheduling of consumer activities: work trips. American Economic Review, 72(3), 467-479.

Smith, M.J. 1979. The existence, uniqueness, and stability of traffic equilibria. Transportation Research Part $B, 13,295-304$.

Wang, J. Y. T., \& Ehrgott, M. 2013. Modelling route choice behaviour in a tolled road network with a time surplus maximisation bi-objective user equilibrium model. Transportation Research Part B, 57, 342-360.

Wang, J. Y. T., Raith, A., \& Ehrgott, M. 2010. Tolling Analysis with Bi-objective Traffic Assignment. Pages 117-129 of: Ehrgott, M., Naujoks, B., Stewart, T., \& Wallenius, J. (eds), Multiple Criteria Decision Making for Sustainable Energy and Transportation Systems. Springer Verlag, Berlin.

Wang, J. Y. T., Ehrgott, M., \& Chen, A. 2014. A Bi-objective User Equilibrium Model of Travel Time Reliability in a Road Network. Transportation Research Part B, 66, 4-15.

Wardrop, J. G. 1952. Some Theoretical Aspects of Road Traffic Research. Proceedings of the Institution of Civil Engineers, Part II, 1, 325-362.

Watling, D. 2006. User equilibrium traffic network assignment with stochastic travel times and late arrival penalty. European Journal of Operational Research, 175, 1539-1556. 